\title{
Excitation and Linear Instability of Stationary Modes in Falkner-Skan-Cooke Boundary Layer
}

\author{
L. Shen and C. $\mathrm{Lu}^{\dagger}$ \\ School of Marine Sciences, Nanjing University of Information Science \& Technology, Nanjing 210044, China \\ †Corresponding Author Email: cglu@nuist.edu.cn
}

(Received December 24, 2020; accepted March 15, 2021)

\begin{abstract}
Past studies showed that a micron-sized surface roughness may cause the generation of a significant unstable, stationary wave in a crossflow boundary layer, and consequently promote or delay the laminar-turbulent transition. The crossflow boundary layer is usually driven by the favorable pressure gradient which is produced by accelerated inviscid velocity. Hence, for a fixed sweep angle, the magnitude of pressure gradient is the key parameter for the excitation and evolution of the stationary crossflow mode. In order to study the effect of pressure gradient on the excitation and subsequent linear development of stationary mode, a classical FalknerSkan-Cooke boundary layer is introduced so that the magnitude of pressure gradient can be easily parameterized by an acceleration coefficient. Numerical simulation is performed to induce the stationary perturbation by chordwise-isolated, spanwise-periodic roughness at the lower branch of neutral curve. Then the excited waves develop into Rayleigh modes in the downstream region. The stationary modes with different spanwise wavenumbers in various favorable-pressure-gradient boundary layers are simulated and analysed to determine the effect of pressure gradient. And the corresponding coupling coefficients are calculated to connect the initial amplitude and the eigenmode of linear stability theory for implementing the existing prediction method of laminar-turbulent transition.
\end{abstract}

Keywords: Stationary wave; Instability; Falkner-Skan-Cooke boundary layer.

\section{INTRODUCTION}

The role of surface roughness of wall-bounded flows is of particular interest in the laminar-turbulent transition. In a crossflow boundary layer, stationary waves with significant amplification can be generated by roughness elements solely (Saric et al. 2003). In the wind-tunnel experiments, even micronsized surface irregularities were able to cause laminar-turbulent transition (Reibert et al. 1996; Carrillo Jr et al. 1997). Radeztsky Jr et al. (1999) changed the height of the roughness, and the measured location of transition moved downstream consequently. Furthermore, the transition is sensitive to the chordwise-isolated, spanwise-periodic roughness elements placed near the attachment line (Radeztsky Jr et al. 1999; Reibert et al. 1996), and an evident influence on the transition has been observed in the experiments which suggests that micron-sized surface roughness can promote or delay the laminar-turbulent transition. By means of spatial direct numerical simulation, Wassermann and Kloker (2002) and Wassermann and Kloker (2003)investigated the transition control strategy of upstream flow deformation (UFD) by discrete roughness elements (DREs) (Saric et al. 2003) and explained the underlying mechanisms for the first time.

For the understanding of the mechanism behind the crossflow-dominated transition, a theoretical analysis must be put forward. Finite-Reynoldsnumber theory (FRNT) was introduced by Crouch (1993) and Choudhari (1994) to study the receptivity of the stationary crossflow modes. It works as solving Orr-Sommerfeld equation with the inhomogeneous boundary condition which represents the wall roughness. The obtained results show a good agreement with the experimental observation (Reibert et al. 1996), though it neglects the non-parallelism effect which plays an important role near the leading edge (Collis and Lele 1999). Complementary to the FRNT, large-Reynolds number asymptotic analysis is carried out by (Choudhari 1994; Choudhari 1995) and (Butler and Wu 2018), to reveal the mechanism of excitation and subsequent linear amplification of so-called longwavelength Rayleigh mode. The crossflow mode is first generated as a wall-shear-aligned mode at the leading order, then develop into a viscous-inviscid interactive regime, and finally become the longwavelength Rayleigh mode. Moreover, the superlinear (Kurz and Kloker 2014) and nonlinear 
response to the roughness height is explained by large-Reynolds number asymptotic analysis as well (Butler and Wu 2018; Choudhari and Duck 1996). Schrader et al. (2009) and Tempelmann et al. (2011) performed direct numerical simulation (DNS) of the generating process of the travelling and stationary crossflow as well. Recently, He et al. (2019) linked the mechanism of laminar-flow-control based on distributed roughness to the resonance between the multiple crossflow modes and the roughnessinduced perturbation in a Fanker-Skan-Cooke boundary layer. The wave-wave interactions in their study include the triadic resonance involving two crossflow eigenmodes and one roughness mode. The generalized resonant mechanism may explain the selected wavenumber of the distributed roughness that is able to delay or promote the transition.

For a fixed sweep angle, the magnitude of pressure gradient is one of the key parameters for the excitation and subsequent linear development of instability. In this paper, a classical Falkner-SkanCooke similarity solution is introduced to study the effect of pressure gradient. Numerical simulation is performed to generate the stationary perturbation by chordwise-isolated, spanwise-periodic roughness. The excited waves are compared with the eigenmodes of linear stability theory and Rayleigh modes in various favorable-pressure-gradient boundary layers. And the corresponding coupling coefficients are calculated for the application of prediction of laminar-turbulent transition.

\section{FORMULATION}

\subsection{Scalings and Base Flow}

We consider a small-height roughness element on the surface of a swept flat plate to induce stationary wave in a classical Falkner-Skan-Cooke Boundary layer as seen in Fig. 1. The simulation is carried out in a Cartesian coordinate system which is nondimensionalized by the displacement thickness of boundary layer $\delta^{*}$ at the inlet boundary of the computational domain,

$$
(x, y, z)=\left(x^{*}, y^{*}, z^{*}\right) / \delta^{*} \text {. }
$$

And the velocities and pressure are nondimensionalized by the far-field chordwise velocity of mean flow $U_{\infty}{ }^{*}$ at the inlet boundary

$$
\left(\begin{array}{c}
u \\
v \\
w
\end{array}\right)=\left(\begin{array}{c}
u^{*} \\
v^{*} \\
w^{*}
\end{array}\right) / U_{\infty}^{*}, \quad p=\frac{p^{*}}{\rho^{*} U_{\infty}^{* 2}},
$$

where $\rho^{*}$ is the reference density. The sweep angle is $\chi=45^{\circ}$. The Reynolds number is defined as $R e=U_{\infty}^{*} \delta^{*} / v^{*}$ with $v^{*}$ being the kinematic viscosity. The Reynolds number at the inlet boundary is set to $R e=180$ which is upstream of
Schrader et al. (2009)'s case $(R e=220)$, so that we can place the roughness element closer to the leading edge of the flat plate. The velocity and pressure are decomposed into two parts, i.e., the base flow and perturbation,

$$
\left(\begin{array}{l}
u \\
p
\end{array}\right)=\left(\begin{array}{l}
U_{B}(x, y) \\
V_{B}(x, y) \\
W_{B}(x, y) \\
P_{B}(x)
\end{array}\right)+h\left(\begin{array}{l}
u(x, y, z, t) \\
v(x, y, z, t) \\
w(x, y, z, t) \\
p(x, y, z, t)
\end{array}\right),
$$

where $h=h^{*} / \delta^{*} \square 1$ is the non-dimensional roughness height that is much smaller than the displacement thickness of boundary layer. The invicid slip velocity of Falkner-Skan-Cooke flow (Shen and Lu 2021b) over the flat plate is

$U_{e}(x)=\left(\frac{x}{x_{0}}\right)^{m}$.

The base flow is assumed to be spanwise uniform. And a similarity variable $\eta=y \sqrt{\operatorname{Re} \frac{U_{e}}{x}}$ and stream function $F(\eta)$ and $G(\eta)$ are introduced so that the base flow velocities can be written as,

$\left\{\begin{array}{l}U_{B}=U_{e} F^{\prime}, \\ V_{B}=\operatorname{Re}^{-1 / 2} \sqrt{\frac{U_{e}}{x}} \frac{\left(F^{\prime} \eta-F\right)-m\left(F+F^{\prime} \eta\right)}{2}, \\ W_{B}=-\tan \chi G .\end{array}\right.$

By substituting the velocities Eq. (5) into the classical boundary layer equations, the governing equations for base flow are obtained

$\left\{\begin{array}{c}F^{\prime \prime \prime}+\frac{1+m}{2} F^{\prime \prime} F+m\left[1-\left(F^{\prime}\right)^{2}\right]=0, \\ G^{\prime \prime}+\frac{1+m}{2} F G^{\prime}=0,\end{array}\right.$

where $m=\frac{x}{U_{e}} \frac{d U_{e}}{d x}$ is the acceleration parameter. We only consider the favorable-pressure-gradient boundary layer, i.e., $m>0$. The lower and upper boundary conditions are

$F=F_{\eta}=G=0$, as $\eta=0$,

and

$F_{\eta}=G=1$, as $\eta \rightarrow \infty$,

respectively.

\subsection{Perturbed flow}

Due to the streamwise-isolated and spanwiseperiodic roughness, the crossflow eigenmodes and roughness-induced perturbations are periodic in the spanwise direction and can be modeled by Fourier components 


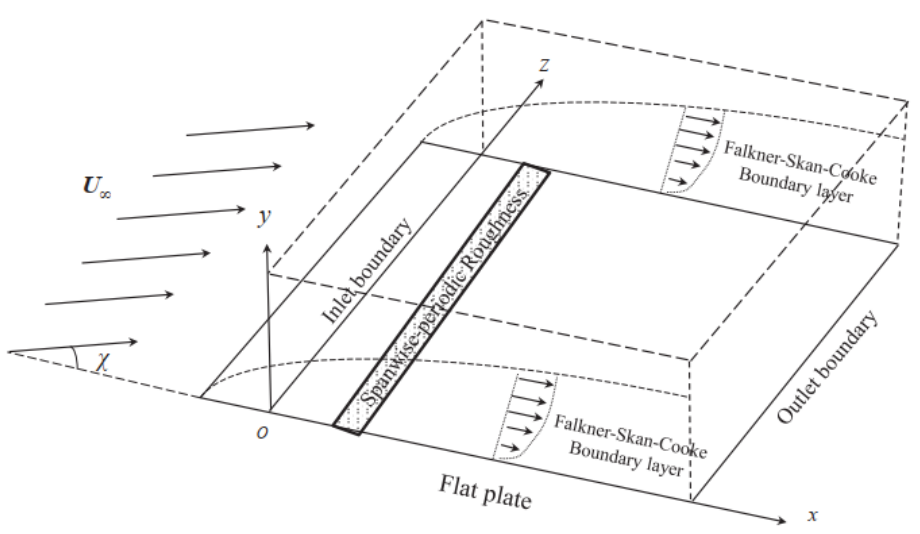

Fig. 1. Sketch of the computational domain.

$\left(\begin{array}{l}u(x, y, z, t) \\ v(x, y, z, t) \\ w(x, y, z, t) \\ p(x, y, z, t)\end{array}\right)=\sum_{n=-M}^{M}\left(\begin{array}{l}\hat{u}_{n}(x, y, t) \\ \hat{v}_{n}(x, y, t) \\ \hat{w}_{n}(x, y, t) \\ \hat{p}_{n}(x, y, t)\end{array}\right) e^{i n k_{3} z}$,

where $k_{3}$ is the fundamental wave number. Substitute Eq. (9) into the perturbed Navier-Stokes equations, then obtain the governing equations for our numerical simulation,

$$
\left\{\begin{array}{c}
\frac{\partial \hat{u}_{n}}{\partial x}+\frac{\partial \hat{v}_{n}}{\partial y}+i \beta_{n} \hat{w}_{n}=0, \\
\ell_{t} \hat{u}_{n}+U_{B, x} \hat{u}_{n}+U_{B, y} \hat{v}_{n}+\mathrm{N} \hat{u}_{n}+\frac{\partial \hat{p}_{n}}{\partial x}=\ell_{R} \hat{u}_{n}, \\
\ell_{t} \hat{v}_{n}+V_{B, x} \hat{u}_{n}+V_{B, y} \hat{v}_{n}+\mathrm{N} \hat{v}_{n}+\frac{\partial \hat{p}_{n}}{\partial y}=\ell_{R} \hat{v}_{n}, \\
\ell_{t} \hat{w}_{n}+W_{B, x} \hat{u}_{n}+W_{B, y} \hat{v}_{n}+\mathrm{N} \hat{w}_{n}+i \beta_{n} \hat{p}_{n}=\ell_{R} \hat{w}_{n},
\end{array}\right.
$$

where the wave number of Fourier component $\beta_{n}=n k_{3} \cdot \ell_{t}=\frac{\partial}{\partial t}+U_{B} \frac{\partial}{\partial x}+V_{B} \frac{\partial}{\partial y}+i \beta_{n} W_{B}$ is the linear convective operator. $\mathrm{N}=h\left(\hat{u}_{n} \frac{\partial}{\partial x}+\hat{v}_{n} \frac{\partial}{\partial y}+i \beta_{n} \hat{w}_{n}\right)$ is the nonlinear operator. And $\ell_{R}=\operatorname{Re}^{-1}\left(\frac{\partial^{2}}{\partial x^{2}}+\frac{\partial^{2}}{\partial y^{2}}-\beta_{n}{ }^{2}\right)$ is the

viscous operator. The equivalent boundary condition for the small surface roughness is expressed as a Maclaurin series of perturbed velocities on the surface,

$$
\left\{\begin{array}{l}
u(x, 0, z)=-U_{B, y}(x, 0) h F(x, z) \\
-U_{B, y y}(x, 0) \frac{h^{2}}{2} F(x, z)^{2}+O\left(h^{3}\right), \\
w(x, 0, z)=-W_{B, y}(x, 0) h F(x, z) \\
-W_{B, y y}(x, 0) \frac{h^{2}}{2} F(x, z)^{2}+O\left(h^{3}\right),
\end{array}\right.
$$

where $F(x, z)$ is shape function of the roughness, and the dimensionless roughness height $\mathrm{h}=0.01$. A smooth top-hat roughness (Kurz and Kloker 2014) is applied here whose contour is defined by a hyperbolic tangent function

$$
F(x, z)=\frac{1}{2}\left[1+\tanh \left(\frac{\frac{d}{2}-\left|x-x_{r}\right|}{\kappa}\right)\right] e^{i \beta z}+c . c .,
$$

where $d$ is the diameter of the roughness element and $\kappa$ controls the maximum slope angle of the element, which is 0.5 in the following computations.

Jaccobi transform is utilized for applying the numerical simulation on non-uniform meshes. Highorder compact schemes (Lele 1992; Wassermann and Kloker 2002; Shen et al. 2019; Shen and Lu 2021a) are used to discretize the spatial derivatives. The fifth-order upwind schemes

$$
\left\{\begin{array}{l}
2 f_{i-1}^{\prime}+3 f_{i}^{\prime}= \\
\frac{-3 f_{i-2}-44 f_{i-1}+36 f_{i}+12 f_{i+1}-f_{i+2}}{12}, \text { for } \quad C_{i}>0, \\
3 f_{i}^{\prime}+2 f_{i+1}^{\prime}= \\
-\frac{-3 f_{i+2}-44 f_{i+1}+36 f_{i}+12 f_{i-1}-f_{i-2}}{12}, \text { for } \quad C_{i}<0,
\end{array}\right.
$$

are used for the convection terms, where $C$ is the propagation speed, $f$ can be any variable in the governing equations. The sixth-order first derivative scheme

$$
\begin{aligned}
& f_{i-1}^{\prime}+3 f_{i}^{\prime}+f_{i+1}^{\prime}= \\
& \frac{7}{3} \cdot\left(f_{i+1}-f_{i-1}\right)+\frac{1}{12}\left(f_{i+2}-f_{i-2}\right),
\end{aligned}
$$

and fifth-order second derivative scheme

$$
\left\{\begin{array}{c}
2 \mathrm{f}_{i-1}^{\prime \prime}+11 \mathrm{f}_{i}^{\prime \prime}+2 \mathrm{f}_{i+1}^{\prime \prime}= \\
\frac{3 f_{i-2}+48 f_{i-1}-102 f_{i}+48 f_{i+1}+3 f_{i+2}}{4},
\end{array}\right.
$$

are used for the pressure gradient term and viscous term respectively. The time marching is implemented by fourth-order Runge-Kutta scheme. Fourth-order iterative schemes are applied to solve the elliptical pressure-divergence equation. The nonlinear terms in the momentum equations are 
solved by the pseudo-spectral method. The computational area is a rectangle space with chordwise length of 800 and normal length of 25 . The perturbed velocities at the inlet boundary is set to zero, while a von Neumann condition is used at the far-field/upper boundary. The wall/lower boundary is applied the non-slip condition unless the roughness area given by Eq. (12), and a non-reflect boundary condition (Jin and Braza 1993) is utilized at the outlet boundary. 800 chordwise-grids and 200 normal-grids is used for the computation. The validation of numerical method and convergence of mesh are shown in section 3 .

\subsection{Eigenvalue modes}

The eigenvalues of the theoretical solutions will be used to identify and analyse the generated stationary mode in the numerical simulation. According to the linear stability theory (LST), the crossflow eigenmodes can take a local normal-modal form

$$
\left(\begin{array}{l}
u(x, y, z) \\
v(x, y, z) \\
w(x, y, z) \\
p(x, y, z)
\end{array}\right)=\left(\begin{array}{c}
\hat{u}(y) \\
\hat{v}(y) \\
\hat{w}(y) \\
\hat{p}(y)
\end{array}\right) e^{i(\alpha x+\beta z),}
$$

where $\alpha=\alpha_{r}+i \alpha_{i}$ is the complex chordwise wavenumber and $\beta$ is the spanwise wavenumber. Substitution of Eq. (16) into linear perturbed NavierStokes equations with parallel-flow assumption leads to

$$
\left\{\begin{array}{c}
i \alpha \hat{u}+D \hat{v}+i \beta \hat{w}=0, \\
i\left(\alpha U_{B}+\beta W_{B}\right) \hat{u}+U_{B, y} \hat{v} \\
=-i \alpha \hat{p}+\operatorname{Re}^{-1}\left(D^{2}-\alpha^{2}-\beta^{2}\right) \hat{u}, \\
i\left(\alpha U_{B}+\beta W_{B}\right) \hat{v} \\
=-D \hat{p}+\operatorname{Re}^{-1}\left(D^{2}-\alpha^{2}-\beta^{2}\right) \hat{v}, \\
i\left(\alpha U_{B}+\beta W_{B}\right) \hat{w}+W_{B, y} \hat{v} \\
=-i \beta \hat{p}+\operatorname{Re}^{-1}\left(D^{2}-\alpha^{2}-\beta^{2}\right) \hat{w},
\end{array}\right.
$$

where $D \equiv \frac{d}{d y}$. By eliminating $u, w$ and $p$, it will be reduced to the steady form of Orr-Sommerfeld equation,

$$
\begin{gathered}
\left\{i \operatorname { R e } \left[\left(\alpha U_{B}+\beta W_{B}\right)\left(D^{2}-\alpha^{2}-\beta^{2}\right)\right.\right. \\
\left.\left.-\left(\alpha U_{B, y y}+\beta W_{B, y y}\right)\right]-\left(D^{2}-\alpha^{2}-\beta^{2}\right)^{2}\right\} \hat{v}=0 .
\end{gathered}
$$

This is an eigenvalue problem by applying the homogeneous boundary conditions.

If the Reynolds number is asymptotic large, the steady Orr-Sommerfeld equation will be reduced to a steady Rayleigh equation,

$$
\begin{gathered}
{\left[\left(\alpha U_{B}+\beta W_{B}\right)\left(D^{2}-\alpha^{2}-\beta^{2}\right)\right.} \\
\left.-\left(\alpha U_{B, y y}+\beta W_{B, y y}\right)\right] \hat{v}=0 .
\end{gathered}
$$

Thus, a critical layer appears, if an inflection point $y_{c}$ exists in the profile of the effective velocity of base flow and satisfy

$$
\left\{\begin{array}{l}
\alpha U_{B}+\beta W_{B}=0, \\
\alpha U_{B, y y}+\beta W_{B, y y}=0,
\end{array} \text { as } y=y_{c},\right.
$$

which can be written as

$$
\frac{U_{B}}{U_{B, y y}}=\frac{W_{B}}{W_{B, y y}} \text { as } y=y_{C}
$$

to find the inflection point of the effective velocity of base flow and the eigenvalue. Obviously, the location of the critical layer is independent of the wavenumber, and the chordwise wavenumber is in a linear relationship with the spanwise wavenmber. In addition, near the leading edge where viscous effect play at the leading order equations, the stationary mode is wall-shear-aligned at the leading order,

$\alpha U_{B, y}+\beta W_{B, y}=0$, as $y=0$.

\section{RESULTS AND DISCUSSION}

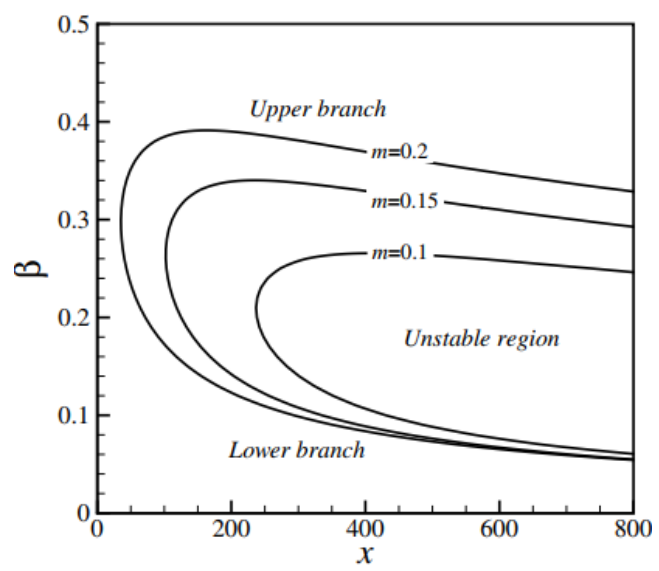

Fig. 2. Neutral curves of base flows with different favorable pressure gradients, $m=0: 1$, 0:15 and 0:2.

Before the numerical simulation, the stability analysis on the base flow is carried out by using linear stability theory Eq. (18). The neutral curves of different favorable pressure gradients are shown in Fig. 2, i.e., $m=0.1,0.15$ and 0.2 . Inside the neutral curve is the unstable region where the perturbed waves are amplifying, whereas outside the neutral curve is the stable region where the perturbed waves are damped. As seen in Fig. 2, the boundary layer of greater acceleration parameter has larger unstable region which begins closer to the leading edge of the flat plate. The higher-spanwise-wavenumber branch of the neutral curve is the so-called upper branch, and the lower-spanwise-wavenumber branch is the lower branch. The most efficient excitation of the stationary wave occurs at the lower branch of the neutral curve, which can experience sufficient amplification once generated. Therefore, the roughness element in this study is placed at the lower 


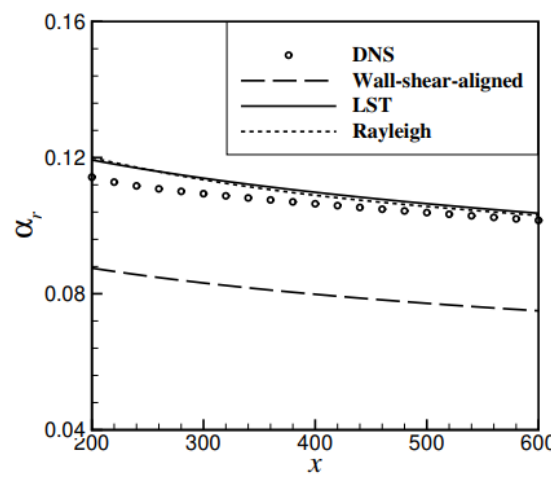

(a) Real part $\alpha_{r}$

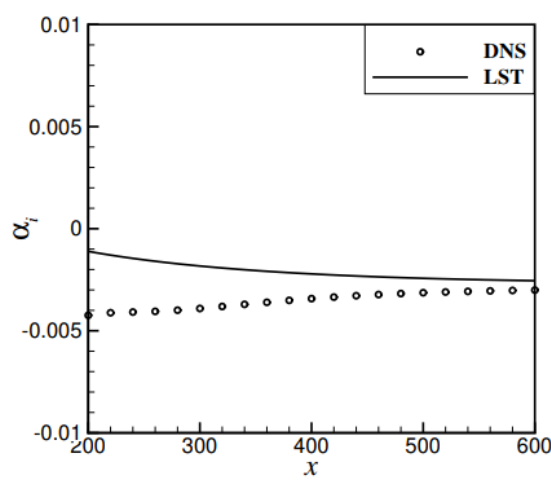

(b) Imaginary part $\alpha_{i}$

Fig. 3. Chordwise evolutions of dispersion relations of the excited stationary wave comparing with those predict by linear stability theory and Rayleigh mode, $m=0.2, \beta=0.16$.

branch for the efficient excitation of the unstable waves.

First, the optimal case of Schrader et al. (2009) is calculated. The base flow is a Falkner-Skan-Cooke Boundary layer of $m=0.2$. The spanwise wavenumber of the wall roughness are chosen to be $\beta=0.16$ and placed at the lower branch. The real part of the chordwise wavenumber $\alpha_{r}$ is associated with the wavelength of the perturbed wave. And its numerical results (Fig. 3a) show a good agreement with the those predicted by linear stability theory and Rayleigh mode as it propagates downstream. The imaginary part of the chordwise wavenumber $\alpha_{i}$ is associated with the amplifying rate (negative values) of the perturbed wave. And its absolute value (Fig. $3 b)$ is larger than those predicted by linear stability theory near the roughness, while both results converge downstream. Moreover, the profiles of the chordwise and spanwise velocity of the excited stationary waves agree well with the eigenfunctions obtained from linear stability theory at $\mathrm{x}=400$ as shown in Fig. 4.

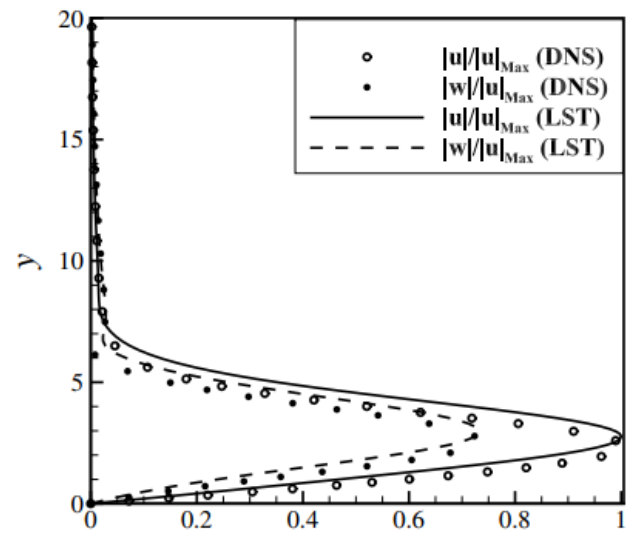

Fig. 4. Profiles of the chordwise and spanwise velocity of stationary wave obtained by numerical simulation comparing with the eigenfunctions computed by linear stability theory at $\mathrm{x}=400, m=0.2, \beta=0.16$.
It indicates that at the sufficient downstream location the excited stationary wave develops into a longwave Rayleigh mode and its dispersion relation match well with the eigenvalue of linear stability theory. Therefore, we can define a coupling coefficient $C_{R}$, namely so-called effective receptivity coefficient (Schrader et al. 2009; Tempelmann et al. 2011), to evaluate the efficiency of the excitation by extrapolating the initial amplitude at the neutral point from the downstream amplitude via the $e^{N}$ method whose amplifying rate $\alpha_{i}$ is computed by linear stability theory.

$C_{R}=\frac{A\left(x_{e}\right)}{e^{N} H\left(\alpha_{r}\right)}, \quad N\left(x_{e}\right)=-\int_{x_{N}}^{x_{e}} \alpha_{i}(x) x d x$,

where $N$ is the integral of the amplifying rate from neutral point $x_{N}$ to downstream location $x_{e}$, and $A\left(x_{e}\right)$ is the downstream amplitude. $H\left(\alpha_{r}\right)$ is the shape function of roughness in Fourier space. In this paper, we choose the amplitude of chordwise velocity $|\mathrm{u}|$ at $x_{e}=700$ as the downstream amplitude and the spanwise velocity has the similar features. The coupling coefficient $C_{R}$ is a constant in the downstream region.

Additionally, the numerical results of the optimal case at $m=0.2$ on meshes of different numbers of $y$-grids $N_{y}$ are presented to verify the numerical method and the convergence of computational mesh. The errors of the dispersion relations of the eigenmodes at downstream location $x=600$ between numerical results on different numbers of grids and theoretical solutions of linear stability theory are shown in Fig. 5. The error of the real part of complex chordwise wavenumber $E\left(\alpha_{r}\right)$ represents the disperse error, and the error of the imaginary part $E\left(\alpha_{i}\right)$ represents the dissipative error. The finer meshes give relatively exact results for both the real part and imaginary part of the wavenumber. And the coarse meshes of less than 150 grids bring in visible errors. In view of this, we 
choose a mesh of $200 y$-grids for maintaining the high accuracy and efficiency of computation.

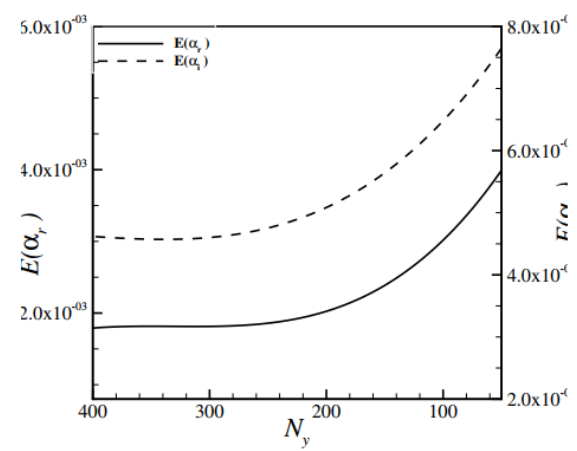

Fig. 5. The errors of the real part $\mathrm{E}\left(\alpha_{r}\right)$ and imaginary part $\mathrm{E}\left(\alpha_{i}\right)$ of complex chordwise wavenumber of the eigenmodes at $x=600$ on different $y$-grid meshes, $m=0: 2, b=0: 16$.

The coupling coefficient $C_{R}$ provides the initial condition for the existing prediction methods of laminar-turbulent transition, for instance, the wellknown $e^{N}$ method based on the linear stability theory. It is calculated by Eq. (23) varying with spanwise wavenumber $\beta$ in different pressuregradient boundary layers and the results are shown in Fig. 6(a). Additionally, as shown in Fig. 6(b), the displacement thickness of boundary layer $\delta_{r}$ at the roughness varying with spanwise wavenumber has a minimum point i.e., $\beta=0.25$ for $m=0.15$ or $\beta=0.3$ for $m=0.2$. That is the location of neutral point as seen in Fig. 2. On the left of the neutral point is the lower branch, whereas on the right of the neutral point is the lower branch. The obtained coupling coefficient of the optimal case ( $\beta=0.16$, $m=0.2$ ) accord well with the numerical result of Schrader et al. (2009). The roughness in our simulation located at the lower branch of neutral curve trigger the worst scenario. As seen in Fig. 6(a), the lower-spanwise-wavenumber stationary waves

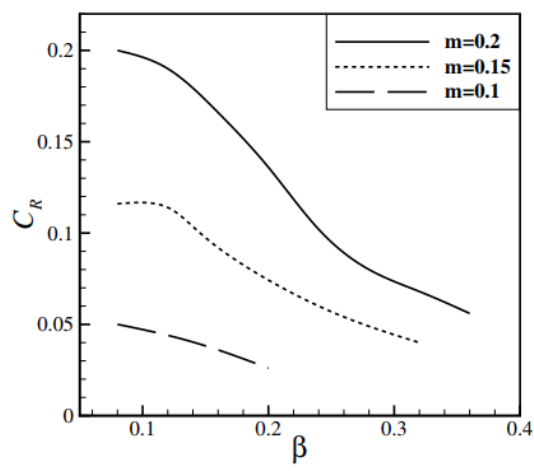

(a) Coupling coefficients $C_{R}$ give higher coupling coefficients for favorablepressure-gradient $m=0.1,0.15$ and 0.2 . It indicates that the lower branch relates to strong excitation. And it also finds the relationship between the excitation and the magnitude of pressure gradient. The greater favorable pressure gradient brings larger coupling coefficients. The $m=0.2$ case has the largest coupling coefficients as seen in Fig. 6(a). It demonstrates that favorable-pressure-gradient promote the exciting process of stationary waves.

The subsequent linear development, after excitation, is important to the entire process of transition as well. The evolution of the dispersion relations of the excited stationary waves in the downstream of roughness are shown in Figs. 7 and 8. Figure 7 gives the real parts of chordwise wavenumbers computed by numerical simulation and linear stability theory. Due to the linear dependence of chordwise wavenumber on the spanwise wavenumber (see Eq. (20)), the results are all normalised by the spanwise wavenumber $\beta$. The dispersion relations of higherspanwise-wavenumber mode ( $\beta=0.24$ ) are closer to the eigenvalues of Rayleigh mode. On the contrary, the lower-spanwise-wavenumber modes $(\beta=0.12,0.16)$ have a smaller chordwise wavenumber than that of Rayleigh mode. It indicates that the viscous effect remains significant in this region. This phenomenon is similar in different pressure-gradient boundary layers $(m=0.15,0.2)$.

Figure 8 gives imaginary parts of chordwise wavenumbers computed by numerical simulation and linear stability theory. Obviously, the amplifying rate has been underestimated by linear stability theory near the roughness. It mainly attributes to the assumption of parallel flow in linear stability theory, which should have non-parallel effect in the leadingedge area. On account of the neutral point of greater favorable-pressure-gradient boundary layer being closer to the leading edge, the corresponding development of stationary mode experiences stronger non-parallelism effect and leads to larger deflection between the results of numerical simulation and linear stability theory as seen the difference in cases $\mathrm{m}=0.2$ and $\mathrm{m}=0.15$.

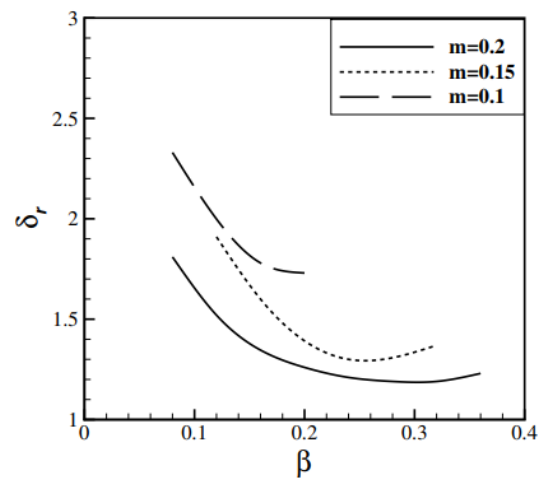

(b) Displacement thickness $\delta_{r}$

Fig. 6. Coupling coefficients $C_{R}$ of stationary waves and displacement thickness of boundary layer $\delta_{r}$ at the roughness varying with spanwise wavenumber in various pressure-gradient boundary layers. 


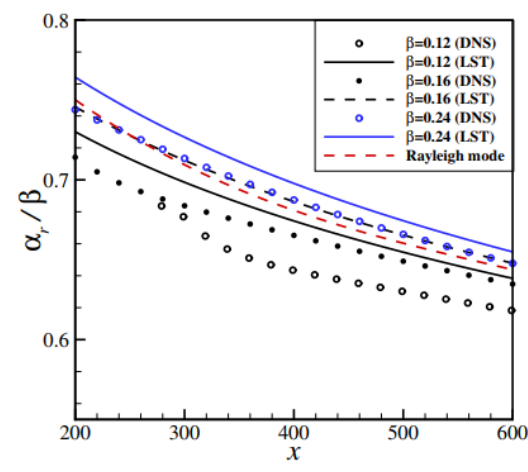

(a) $m=0.2$

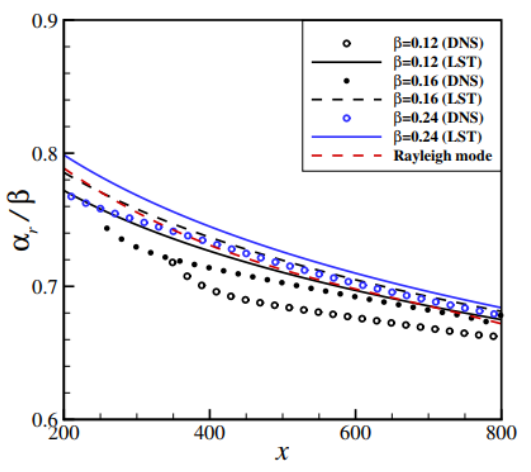

(b) $m=0.15$

Fig. 7. Real parts of chordwise wavenumbers $\alpha_{r}$ computed by numerical simulation and linear stability theory comparing with the eigenvalue of Rayleigh mode.

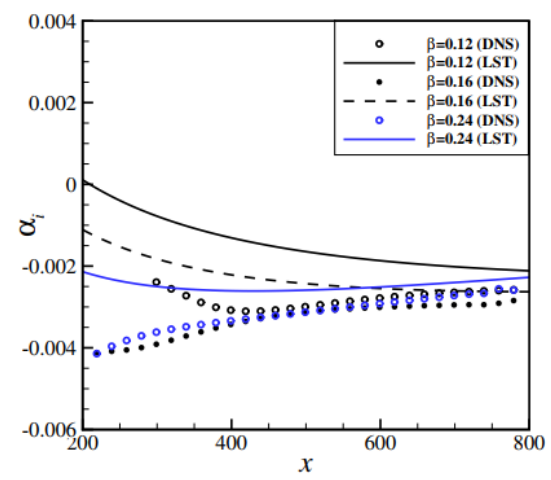

(a) $m=0.2$

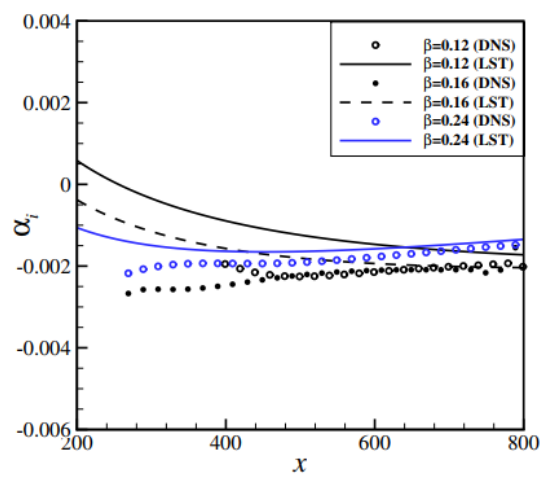

(b) $m=0.15$

Fig. 8. Imaginary parts of chordwise wavenumbers $\alpha_{i}$ computed by numerical simulation and linear stability theory.

According to Eq. (23), the larger receptivity coefficients of lower-spanwise-wavenumber modes (Fig. 6) are partly due to the relatively smaller amplification rates (Fig. 8). Because, even if the unstable waves have the equal downstream amplitude, the smaller amplification rates would obtain larger receptivity coefficients.

Nonetheless, the amplifying rates of numerical simulation and linear stability theory converge in the downstream. It suggests that the coupling coefficient, which is proposed to connect the initial amplitude and the eigenmode of linear stability theory, can be applied to the prediction method of laminar-turbulent transition as the initial conditions in different pressure-gradient boundary layers ( $\mathrm{Xu}$ et al. 2016). The computation of $e^{N}$ method is much more economical than the cost of DNS which means this application in the prediction of transition will be highly valuable.

\section{CONCLUSIONS}

In this paper, we simulate the excitation and subsequent development of the stationary waves in the Falkner-Skan-Cooke boundary layers numerically. The surface roughness is placed at the lower branch of neutral curve to induce the instability efficiently. The obtained results have been compared with the eigenmodes of linear stability and Rayleigh modes. The dispersion relations of higherspanwise-wavenumber waves match well with the eigenvalues of inviscid Rayleigh mode. On the contrary, the lower-spanwise-wavenumber waves have a relatively smaller chordwise wavenumber which indicates that the viscous effect remains significant in this region.

The neutral point of greater favorable-pressuregradient boundary layer is closer to the leading edge, hence the corresponding development experiences stronger non-parallelism effect and leads to larger deflection between numerical results and linear stability theory. Nonetheless, the amplifying rates of numerical simulation and linear stability theory still converge in the downstream where non-parallelism effect fades out. It suggests that the coupling coefficient, which is proposed to connect the initial amplitude and the eigenmode of linear stability theory, can be applied to the prediction method of laminar-turbulent transition as the initial conditions. 
The numerical results show that lower-spanwisewavenumber roughness introduces higher coupling coefficient. And the greater favorable-pressuregradient bring larger coupling coefficient. It demonstrates that favorable-pressure-gradient promote the exciting process of stationary waves.

\section{ACKNOWLEDGMENTS}

This project is supported by the National Natural Science Foundation of China (No.l,12072164) and the Natural Science Foundation of Jiangsu Province, China (No.\,BK20180781).

\section{REFERENCES}

Butler, A. and X. Wu (2018). Stationary crossflow vortices near the leading edge of threedimensional boundary layers: the role of non-parallelism and excitation by surface roughness. Journal of Fluid Mechanics 845, 93.

Carrillo Jr, R. B., M. S. Reibert and W. S. Saric (1997). Distributed-roughness effects on stability and transition in swept-wing boundary layers.

Choudhari, M. (1994). Roughness-induced generation of crossflow vortices in threedimensional boundary layers. Theoretical and Computational Fluid Dynamics 6(1), 1-30.

Choudhari, M. (1995). Long-wavelength asymptotics of unstable crossflow modes, including the effect of surface curvature. Proceedings of the Royal Society of London. Series A: Mathematical and Physical Sciences 451(1943), 515-541.

Choudhari, M. and P. W. Duck (1996). Nonlinear excitation of inviscid stationary vortex in a boundary-layer flow.

Collis, S. S. and S. K. Lele (1999). Receptivity to surface roughness near a swept leading edge. Journal of Fluid Mechanics 380, 141-168.

Crouch, J. (1993). Receptivity of threedimensional boundary layers. In 31st Aerospace Sciences Meeting, pp. 74.

He, J., A. Butler and X. Wu (2019). Effects of distributed roughness on crossflow instability through generalized resonance mechanisms. Journal of Fluid Mechanics $858,787-831$.

Jin, G. and M. Braza (1993). A nonreflecting outlet boundary condition for incompressible unsteady navier-stokes calculations. Journal of computational physics 107(2), 239-253.

Kurz, H. B. and M. J. Kloker (2014). Receptivity of a swept-wing boundary layer to micronsized discrete roughness elements. Journal of fluid mechanics 755, 62.

Lele, S. K. (1992). Compact finite difference schemes with spectral-like resolution. Journal of computational physics 103(1), 16-42.

Radeztsky Jr, R. H., M. S. Reibert and W. S. Saric (1999). Effect of isolated micronsized roughness on transition in sweptwing flows. AIAA journal 37(11), 13701377.

Reibert, M., W. Saric, R. Carrillo, Jr and K. Chapman (1996). Experiments in nonlinear saturation of stationary crossflow vortices in a swept-wing boundary layer. In 34th Aerospace Sciences Meeting and Exhibit, pp. 184.

Saric, W. S., H. L. Reed and E. B. White (2003). Stability and transition of threedimensional boundary layers. Annual Review of Fluid Mechanics 35(1), 413440.

Schrader, L.-U., L. Brandt and D. S. Henningson (2009). Receptivity mechanisms in three-dimensional boundary-layer flows. Journal of Fluid Mechanics 618(1), 209-241.

Shen, L. and C. Lu (2021a). Effect of leadingedge curvature on receptivity of stationary cross-flow modes in swept-plate boundary layers. Advances in Applied Mathematics and Mechanics 13(2), 467480.

Shen, L. and C. Lu (2021b). Effect of pressure gradient on local excitation of boundarylayer instability due to freestream turbulence and micro surface roughness. Journal of Applied Fluid Mechanics 14(4).

Shen, L., C. Lu and X. Zhu (2019). Leadingedge receptivity of boundary layer to threedimensional free-stream turbulence. Applied Mathematics and Mechanics 40(6), 851-860.

Tempelmann, D., L. U. Schrader, A. Hanifi, L. Brandt and D. Henningson (2011). Sweptwing boundary-layer receptivity to localised surface roughness. Journal of Fluid Mechanics, 711. 516-544. 
L. Shen and C. Lu / JAFM, Vol. 14, No. 5, pp. 1437-1445, 2021.

Wassermann, P. and M. Kloker (2002). Mechanisms and passive control of crossflow-vortex-induced transition in a three-dimensional boundary layer. Journal of Fluid Mechanics 456, 49.

Wassermann, P. and M. Kloker (2003). Transition mechanisms induced by travelling crossflow vortices in a three- dimensional boundary layer. Journal of Fluid Mechanics 483, 67.

Xu, J., J. Bai, L. Qiao and Y. Zhang (2016). Correlation-based transition transport modeling for simulating crossflow instabilities. Journal of Applied Fluid Mechanics 9(5). 